\title{
A modern POPMUSIC matheuristic for the capacitated vehicle routing problem
}

\author{
Eduardo Queiroga*1, Ruslan Sadykov ${ }^{\dagger 2}$, and Eduardo Uchoa ${ }^{\ddagger 3}$ \\ ${ }^{1}$ Instituto de Computação, Universidade Federal Fluminense, Av. Gal. Milton \\ Tavares de Souza, s/n, São Domingos, 24210-346, Niterói, Brazil \\ ${ }^{2}$ INRIA Bordeaux, Sud-Ouest, 200 Avenue de la Veille Tour, 33405 Talence, \\ France \\ ${ }^{3}$ Departamento de Engenharia de Produção, Universidade Federal Fluminense, \\ Rua Passo da Pátria 156, Niterói, Brazil
}

November 7, 2020

\begin{abstract}
This work proposes a partial optimization metaheuristic under special intensification conditions (POPMUSIC) for the classical capacitated vehicle routing problem (CVRP). The proposed approach uses a branch-cut-and-price algorithm as a powerful heuristic to solve subproblems whose dimensions are typically between 25 and 200 customers. The whole algorithm can be seen as the application of local search over very large neighborhoods, starting from a single initial solution. The main computational experiments were carried out on instances having between 302 and 1000 customers. Using initial solutions generated by some of the best available metaheuristics for the problem, POPMUSIC was able to obtain consistently better solutions for long runs of up to 32 hours. In a final experiment, starting from the best known solutions available in CVRP library (CVRPLIB), POPMUSIC was able to find new best solutions for several instances, including some very large ones.
\end{abstract}

\section{Introduction}

The Capacitated Vehicle Routing Problem (CVRP), introduced by Dantzig and Ramser (1959), is one of the most widely studied problems in combinatorial optimization and operations research. The CVRP is the prototypical vehicle routing problem. New ideas are often first proposed and tested on CVRP and then generalized to other routing variants. It can be defined as follows. Let $G=(V, E)$ be a complete undirected graph, such that $V=\{0,1, \ldots, n\}$ is the set of vertices and $E$ is the set of edges, where vertex 0 represents a depot and $V_{+}=\{1, \ldots, n\}$ a set of customers. There is a non-negative cost $c_{i j}$ for each edge $\{i, j\} \in E$ and a demand $d_{i}$ for each customer $i \in V_{+}$. The vehicle capacity is denoted by $Q$. A route is a path that begins and ends at the depot. A solution consists of a set of routes that respect the following constraints: (i) each customer must be visited exactly once by one of the routes; (ii) the sum of the customer demands in a route can not exceed the vehicle capacity. The objective is to find a set of routes with the minimum total cost.

Given that CVRP is NP-hard, most of the algorithms proposed for this problem are heuristics and metaheuristics (Laporte et al., 2014). The best performing published algorithms are: the

\footnotetext{
*eduardoqueiroga@id.uff.br

†ruslan.sadykov@inria.fr

‡uchoa@producao.uff.br
} 
iterated local search with set partitioning (ILS-SP) (Subramanian et al., 2013), knowledge-guided local search (KGLS) (Arnold and Sörensen, 2019), hybrid genetic search (HGS) (Vidal et al., 2012), slack induction by string removals (SISR) (Christiaens and Vanden Berghe, 2020), and fast ILS localized optimization (FILO) (Accorsi and Vigo, 2020). ILS-SP combines the wellknown ILS (Lourenço et al., 2019) with a set partitioning (SP) model. The SP model attempts to build unexplored solutions from the set of routes associated with the local minima found by previous runs of the local search. KGLS presents an efficient GLS with three complementary operators using ideas from sequential search and pruning, as well as a problem-specific knowledge to penalize "bad" edges. HGS is a population-based evolutionary search that also makes use of local search (in a step called education) and a sophisticated mechanism for controlling population diversity. Among the key components of HGS, we can mention the management of a subpopulation with infeasible solutions, as well as the individual evaluation (a.k.a. fitness) driven by the solution cost and its contribution to population diversity. SIRS is a ruin 8 recreate local search guided by simulated annealing (SA) (Kirkpatrick et al., 1983). The ruin procedure removes strings (sequence of consecutive customers) from routes (inducing a capacity slack), whereas the recreate procedure reinserts the removed customers in the ruined solution in a greedy manner. Finally, FILO is a scalable metaheuristic that employs novel and existing acceleration techniques during the main iterative part based on ILS, whereas it uses an SA-based acceptance criterion to get a continuous diversification.

On the other hand, the exact methods for CVRP have advanced considerably in recent years (Poggi and Uchoa, 2014; Costa et al., 2019). The state-of-the-art results are achieved by branchcut-and-price algorithms (Pecin et al., 2014, 2017a; Pessoa et al., 2020), which combine column and cut generation with several additional mechanisms. According to the experiments carried out in Uchoa et al. (2017), this type of algorithm is able to produce optimal solutions for almost all instances with up to 250 customers, and in some cases, it can solve even larger instances (the largest one already solved has 654 customers). An important observation on the behavior of modern branch-cut-and-price algorithms for CVRP, explored in this work, is the following: while instances with more than 200 customers usually take hours or even days to be solved, many instances with up to 150 customers can be solved in few minutes, and many instances with up to 100 customers can be solved in seconds.

The algorithms that hybridize metaheuristics with mathematical programming approaches (Jourdan et al., 2009) are often known as matheuristics. Such methods have already been proposed for several optimization problems, including vehicle routing (Archetti and Speranza, 2014; Leggieri and Haouari, 2018). According to Archetti and Speranza (2014), one of the types of matheuristics is based on the decomposition of the original problem into smaller subproblems that can be solved (optimally or sub-optimally) through mathematical programming models. This work proposes a simple Partial OPtimization Metaheuristic Under Special Intensification Conditions (POPMUSIC) (Taillard and Voss, 2002) for the CVRP that uses a modern branchcut-and-price algorithm to solve subproblems (exactly or heuristically). The general idea of POPMUSIC is to optimize subproblems, defined by parts of a solution until a local minimum is reached. This type of algorithm has been shown to be effective for different problems (Taillard and Voss, 2002), including vehicle routing variants (Ostertag et al., 2009; Lalla-Ruiz and Voß, 2020) and the famous traveling salesman problem (Taillard and Helsgaun, 2019).

The remainder of this paper is organized as follows. In Section 2, the proposed POPMUSIC matheuristic for the CVRP is presented. Section 3 describes the modifications to the published branch-cut-and-price algorithm used for solving the subproblems. Section 4 presents and analyses the results of extensive computational experiments. Finally, in Section 5, the final conclusions are presented, as well as suggestions for future work.

\section{A POPMUSIC matheuristic for the CVRP}

Algorithm 1 shows the pseudocode of the proposed POPMUSIC matheuristic for the CVRP, which has four inputs: (i) an initial solution $S$; (ii) an algorithm $\mathcal{A}$ to solve subproblems; 
(iii) initial value $\alpha$ for the current target dimension $\operatorname{dim}_{s p}$ (upper limit on the dimension of subproblems); (iv) step size $\delta$ to increase $\operatorname{dim}_{s p}$. The algorithm's output is a (possibly) improved solution $S$ obtained after solving a sequence of subproblems. A solution $S$ is a set $\left\{r_{1}, \ldots, r_{m}\right\}$ of $m$ routes, whereas the set of customers visited by a route $r$ is denoted by $C(r)$. A set $V_{s p} \subseteq V_{+}$ represents the CVRP subproblem associated with the subgraph $G\left[\{0\} \cup V_{s p}\right]$. We will refer to solutions for subproblems as subsolutions. In addition, in the description of the algorithm, we will consider that $c_{j i}=c_{i j}, \forall\{i, j\} \in E$, and $c_{i i}=0, \forall i \in V_{+}$.

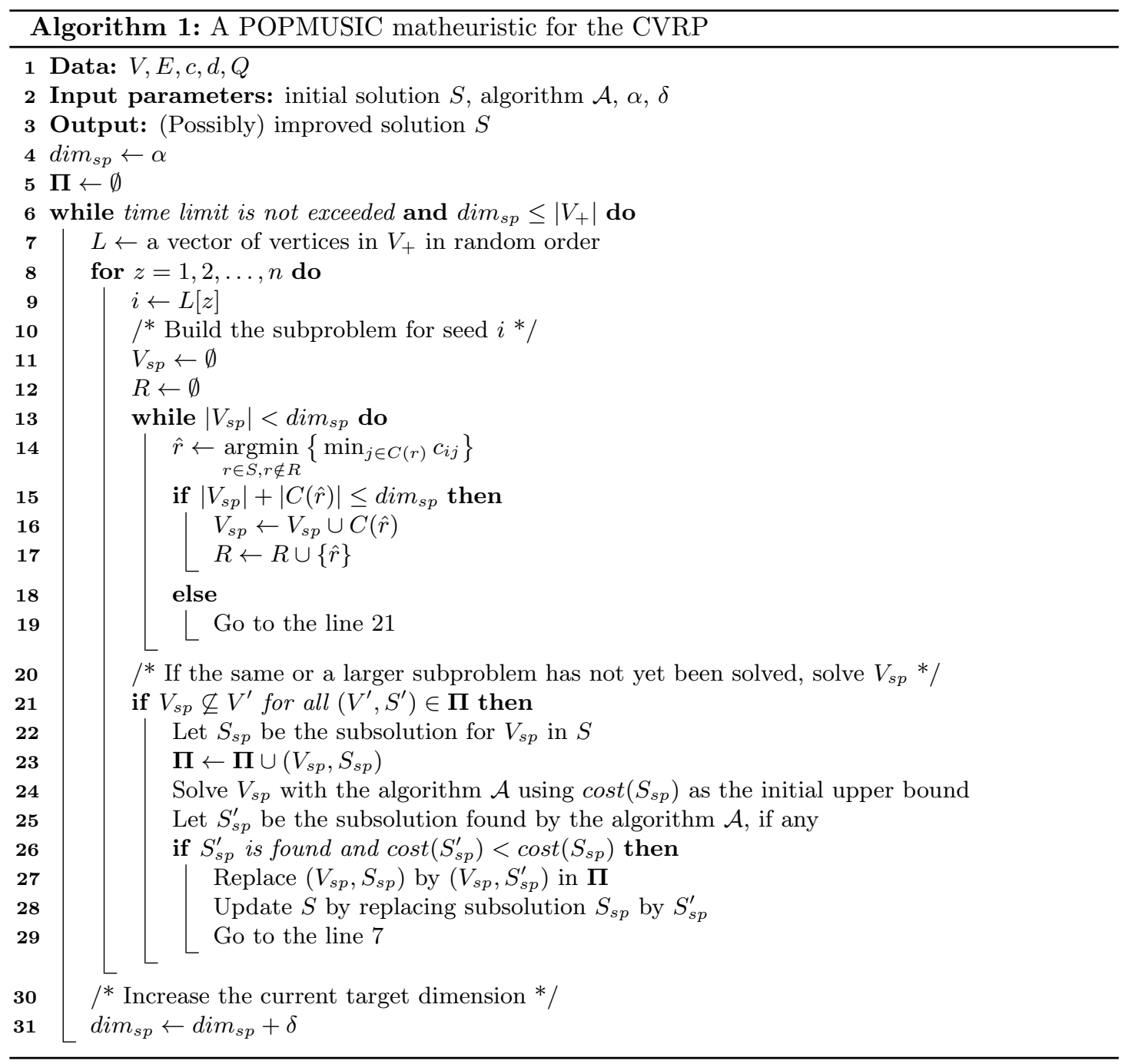

The algorithm keeps the current target dimension $\operatorname{dim}_{s p}$, which is the upper limit for $\left|V_{s p}\right|$. $\operatorname{dim}_{s p}$ is initialized to $\alpha$ at line 4 . The set $\boldsymbol{\Pi}$, initialized at line 5 , keeps all subproblems already explored during the search together with their subsolutions. Formally, $\boldsymbol{\Pi}$ is a set of all pairs $\left(V^{\prime}, S^{\prime}\right)$, such that subproblem with set $V^{\prime} \subset V_{+}$of vertices is already solved, and $S^{\prime}$ is its subsolution. At first, a random permutation of the customers in $V_{+}$produces the array $L$ (line 7 ). For a given value of $d_{i m}$, each customer $i \in V_{+}$is used as a seed to construct a subproblem $V_{s p}$ at lines 11-19. A subproblem $V_{s p}$ with at most $\operatorname{dim}_{s p}$ customers is constructed iteratively by including the routes in the current solution $S$ that are closest to vertex $i$. The distance from $i$ to each route $r \in S$ is determined by the smallest cost of edges connecting $i$ and the vertices in $r$ (line 14). The routes already included in $V_{s p}$ are stored in $R$ to avoid repetitions (line 17). The construction of subproblem $V_{s p}$ is finished when the next selected route $\hat{r}$ cannot be included in subproblem due to the upper limit $\operatorname{dim}_{s p}$ on the subprobem dimension (line 19). Figure 1a illustrates the construction of the subproblem for an instance having 109 customers 
and the current target dimension $\operatorname{dim}_{s p}=30$. First, the route containing the seed (in black) is included in the subproblem. The second selected route is the red one, while the third is the blue one, and the fourth is the purple one. Adding a fifth route would exceed $\operatorname{dim}_{s p}$, so the obtained subproblem has 24 customers.


(a) Initial solution and a constructed subproblem.(b) Improved solution after finding a better subsoSeed customer is marked in black. lution

Figure 1: Constructing and solving a subproblem. Depot is the yellow square, and customers are circles with diameter proportional to its demand. For the sake of visualization, the edges adjacent to the depot are not depicted.

The algorithm solves generated subproblem $V_{s p}$ only if it is neither equal nor contained in any subproblem $V^{\prime}$ already solved before (line 21 ). Indeed, the $\Pi$-based condition avoids wasting time on subproblems, i.e., current subsolutions of which are unlikely to be improved because the same or a larger subproblem has been solved already. The solved subproblems together with their solutions are added to set $\boldsymbol{\Pi}$ at line 23. At line 24 , the algorithm $\mathcal{A}$ tries to improve the subsolution $S_{s p}$ of $S$ for current subproblem $V_{s p}$. As algorithm $\mathcal{A}$, we use a branch-cutand-price based heuristic described in Section 3. It is important here to use the cost of the known solution $S_{s p}$ for subproblem $V_{s p}$ to improve the performance of the branch-cut-and-price algorithm. Finally, if the solution $S_{s p}^{\prime}$ found by $\mathcal{A}$ is better than $S_{s p}$, then $S$ is updated, and the search is restarted for the same target dimension $\operatorname{dim}_{s p}$ : all customers will be used again as seeds without increasing $\operatorname{dim}_{s p}$. Figure $1 \mathrm{~b}$ depicts an example of such an improved solution. If all seeds fail to produce an improving subsolution, then the target dimension $\operatorname{dim}_{s p}$ is increased by $\delta$, so that larger subproblems can be explored (line 31). The algorithm is interrupted when the time limit is reached or when the target dimension exceeds the number of customers (line 6). From now on, we refer to Algorithm 1 as POP.

\section{A branch-cut-and-price heuristic to solve subproblems}

The algorithm $\mathcal{A}$ in POP, used for solving the subproblems, is an adaptation of the generic Branch-Cut-and-Price (BCP) algorithm proposed by Pessoa et al. (2020), which is a state-ofthe-art exact algorithm for many VRP variants, including the CVRP. BCP is a well-known technique that incorporates column and cut generation in a branch-and-bound procedure. In particular, the BCP by Pessoa et al. (2020) includes advanced elements, such as: (i) ng-path relaxation (Baldacci et al., 2011); (ii) rank-1 cuts with limited memory (Jepsen et al., 2008; Pecin 
et al., 2014, 2017b; Bulhões et al., 2018); (iii) path enumeration (Baldacci et al., 2008; Contardo and Martinelli, 2014); (iv) rounded capacity cuts (Laporte and Nobert, 1983); (v) bucket graph based bi-directional labeling algorithm (Sadykov et al., 2020); (vi) edge elimination based on reduced costs (Irnich et al., 2010; Sadykov et al., 2020). The reader is referred to Pessoa et al. (2020) for more details about the BCP algorithm.

Since the methodology proposed in this work is a matheuristic one, optimality does not need to be preserved by the BCP. Thus, we turn the BCP algorithm into a heuristic (named $\mathrm{BCP}_{H}$ ) by:

- Imposing a branch-and-bound node limit of 10 and time limit of 3,600 seconds;

- Using the false gap mechanism, described next;

- Using a restricted master heuristic, described below.

As mentioned above, the BCP algorithm uses an elimination procedure that removes edges from graph $G$ by exploiting reduced cost arguments. In particular, if the minimum reduced cost of a path passing by an edge $e \in E$ is not smaller than the gap between the current upper bound and the lower bound obtained by the column generation procedure, then edge $e$ can safely be removed from the graph $G$, as no improving solution contains this edge. Removing edges makes subsequent calls to the labeling algorithm used for solving the pricing problem faster.

In addition to the edge elimination, path enumeration is also dependent on the gap. This procedure tries to enumerate all possible paths with reduced cost smaller than the current gap between upper and lower bounds. If path enumeration is successful (i.e., the number of enumerated paths is less than, say, one million; enough to store them in a table), the pricing problem from now on is solved by inspection. The inspection of enumerated paths is usually much faster to perform than to call the labeling algorithm. If the number of enumerated routes is sufficiently small (less than 10,000), the current node in the search tree can be finished by adding all enumerated routes to the restricted master and solving it as an IP (using a general solver like CPLEX).

The previous two paragraphs show the importance of having very good upper bounds (and, therefore, smaller gaps) for reducing the running time of the BCP algorithm. In fact, that is why POP solves smaller subproblems first (easy even with not so good upper bounds), so the solution of larger subproblems can benefit from already improved upper bounds. To further reduce the running time, the false gap mechanism artificially decreases the gaps when performing edge elimination and route enumeration. The false gap is defined as $F G=(U B-L B) / F G F$, where the false gap factor $F G F>1$ is a parameter. Application of the false gap mechanism can result in removing edges or paths which participate in an improving solution. However, experiments indicate that such an outcome occurs rarely when one uses a moderate value for $F G F$ (we tested $F G F=3)$.

Another difference from the default BCP algorithm by Pessoa et al. (2020) consists in using an additional heuristic (similar to the one proposed in Pessoa et al. (2009)). It is called after the convergence of column and cut generation at every node of the search tree. The idea is to further decrease the false gap (dividing it by two in each iteration) until it is possible to complete the path enumeration. Then, the 10,000 routes with smaller reduced costs are used to create an IP that is solved by CPLEX.

\section{Computational experiments}

The proposed algorithm POP was coded in Julia language version 1.4.2. The algorithm $\mathrm{BCP}_{H}$ to solve subproblems was obtained by parameterizing the CVRP demo application of VRPSolver (Bulhões et al., 2020). The parameters are described in Section 4.3. VRPSolver, freely available for academic use, implements the generic BCP algorithm proposed by Pessoa et al. (2020). It 
makes use of the BaPCod C++ library (Vanderbeck et al., 2018) as a BCP framework combined with the $\mathrm{C}++$ implementations by (Sadykov et al., 2020) for solving pricing problems, route enumeration, and separation of rank-1 cuts. It also uses CVRPSEP package (Lysgaard, 2003) for separating rounded capacity cuts. Finally, VRPSolver uses CPLEX 12.9 to solve the LP relaxations and the MIPs over the enumerated paths.

All experiments with POP were performed on a 2 Deca-core Haswell Intel Xeon E5-2680 v3 server with $2.50 \mathrm{GHz}$ and $128 \mathrm{~GB}$ of RAM, where each algorithm was executed on a single thread for each instance. Parallel runs for several different instances were performed on the same machine to speed up the experiments, effectively reducing the amount of memory allocated to each process.

\subsection{Benchmark instances}

The tests were performed on the 57 largest instances of the benchmark set X (Uchoa et al., 2017), ranging from 303 to 1001 vertices. Indeed, set X is currently the main benchmark used to assess the performance of all recent exact and heuristic algorithms for the CVRP. We skipped the 43 instances with less than 300 customers because most of those instances are now relatively easy for modern heuristics and even for modern exact algorithms. In fact, 39 of them have proved optimal solutions.

For a deeper analysis of some experiments, we split the 57 instances into two subsets: the subset $\mathrm{X}_{S}$ of 29 instances with $n / K_{\min } \leq 10.8$ (i.e., instances with short routes), and the subset $\mathrm{X}_{L}$ composed by the other 28 instances (i.e., instances with long routes). The $K_{m i n}$ value is an instance attribute that means the minimum possible number of routes that a solution can have. For example, the instance X-n561-k42 belongs to $\mathrm{X}_{L}$ because $n / K_{\min }=561 / 42=13.6>10.8$. Extensive experiments presented in Pecin et al. (2017a) indicate that modern branch-cut-andprice algorithms for CVRP, like the one we use to solve subproblems in POP, perform considerably better on instances with shorter routes. Therefore, route size is a factor that is likely to affect the overall performance of POP.

Moreover, in the preliminary experiments used for calibration, we consider a small representative subset $\mathrm{X}_{R}$ having only seven instances. The choice of $\mathrm{X}_{R}$ is described in $\mathrm{A}$.

The gap of a solution $S$ is calculated as $100 \cdot((\operatorname{cost}(S)-\mathrm{BKS}) / \mathrm{BKS})$, where BKS is the best known solution in the CVRPLIB ${ }^{1}$, only disregarding the solutions found by executions based on the proposed POP approach. Several optimization groups compete for improving the best known solutions for the instances in CVRPLIB. In fact, there were 24 updates in 2020 by seven distinct groups. Updating a BKS in CVRPLIB does not require the publication of an article; one only has to send the improved solution to be checked, even if the improvement is by only one unit. It is not necessary to describe how the solution was obtained. The competing groups may perform long runs of their methods, try several random number seeds, and even resort to special calibration. Thus, those BKSs are likely to be very close to optimum values.

\subsection{Obtaining an initial solution}

The initialization of POP is a critical issue. Preliminary experiments showed that it did not work so well as a stand-alone algorithm. It means that if it is initialized with a low-quality solution $S$ obtained by a simple constructive heuristic, the overall performance of POP is not competitive with the best existing heuristics. In fact, we are proposing POP essentially as an effective way of improving solutions that are already reasonably good, possibly obtained by running some metaheuristic.

We report results obtained by different variants of $\mathrm{POP}^{1}$, which uses the HGS metaheuristic by Vidal et al. (2012) to obtain the initial solution. However, as shown in B, the HGS is more effective if the entire algorithm is restarted (with a different random number seed) after 50,000

${ }^{1}$ BKSs available in the CVRP Library (http://vrp.atd-lab.inf.puc-rio.br/) on October 31, 2020 
iterations without any improvement (a method hereafter called $\operatorname{HGS}^{r}$ ). Notation $\mathrm{POP}_{t}^{1}$ defines the variant that starts POP with the solution obtained by $\mathrm{HGS}^{r}$ in $t$ hours. We tested 4 values for $t$ : 0.01 (36 seconds), 0.125 (450 seconds), 0.5 (1800 seconds), and 2 (7,200 seconds). Of course, the initialization time is included in the overall time. For example, in variant $\mathrm{POP}_{0.5}^{1}$, which is run for 32 hours, $\operatorname{HGS}^{r}$ obtains the initial solution in 0.5 hours, and then POP spends 31.5 hours improving the initial solution.

The results obtained by several variants $\mathrm{POP}^{1}$ over the time horizon of 32 hours are compared with those by $\mathrm{HGS}^{r}$ itself. We also perform some comparisons with a second metaheuristic, the ILS-SP proposed by Subramanian et al. (2013). As shown in Uchoa et al. (2017), although the HGS is on average substantially better than the ILS-SP, there are some instances (usually those with very short routes) where the ILS-SP is superior.

\subsection{Parameterization of the subproblem solver}

The default parameterization of the VRPSolver CVRP demo is calibrated to find optimal solutions for hard instances having around 200-300 customers. As POP needs to solve many smaller problems, we propose an alternative parameterization that works better inside POP. C presents the default and the proposed parameterizations, whose performances are compared in Figure 2 by running $\mathrm{POP}_{0.5}^{1}$ on the $\mathrm{X}_{R}$ instances over 8 hours. The convergence curves of the algorithms show the average gap found at different times.

The figure shows the superior performance obtained when the heuristic version $\mathrm{BCP}_{H}$ is used to solve the subproblems. $\mathrm{BCP}_{H}$ is obtained from the exact $\mathrm{BCP}$ using the proposed parameterization and by setting three additional parameters: RCSPfalseGapFactor to 3 (this activates the false gap mechanism described in Section 3), MaxNbOfBBtreeNodeTreated to 10 (maximum number of nodes in the branch-and-bound tree), and GlobalTimeLimit to 3600 seconds (maximum time for solving a subproblem) in the proposed parameterization. All POP results hereafter are obtained using algorithm $\mathrm{BCP}_{H}$ as the subproblem solver.

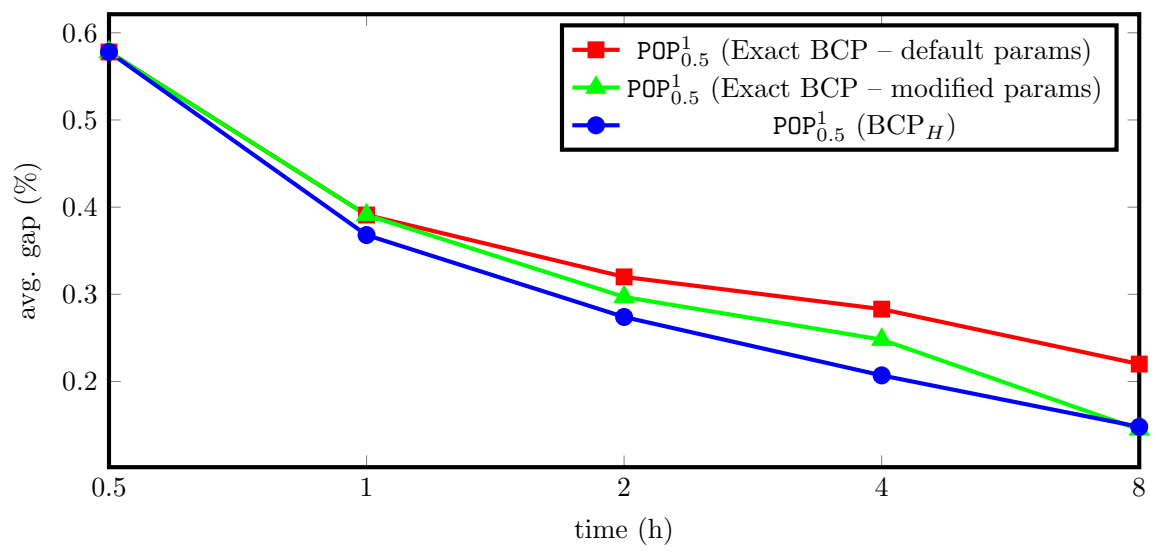

Figure 2: $\mathrm{POP}_{0.5}^{1}$ with three different parameterizations of VRPSolver. The time axis is on a $\log _{2}$ scale.

\subsection{Calibrating parameters $\alpha$ and $\delta$}

Table 1 shows the performance of $\mathrm{POP}_{0.5}^{1}$ for different values of parameters $\alpha$ and $\delta$. Each setting was applied to the $\mathrm{X}_{R}$ instances over the horizon of 8 hours. The setting $(\alpha=50, \delta=40)$ achieved the best performance for 2,4 , and 8 hours. Therefore, all POP results below are obtained with parameterization $(\alpha=50, \delta=40)$. 
Table 1: Avg. gap (\%) of $\mathrm{POP}_{0.5}^{1}$ on $\mathrm{X}_{R}$ instances for different values of $\alpha$ and $\delta$.

\begin{tabular}{cccccccccc}
\hline \multirow{2}{*}{ Time $(\mathrm{h})$} & $\alpha=25$ & $\alpha=25$ & $\alpha=25$ & $\alpha=50$ & $\alpha=50$ & $\alpha=50$ & $\alpha=75$ & $\alpha=75$ & $\alpha=75$ \\
& $\delta=10$ & $\delta=25$ & $\delta=40$ & $\delta=10$ & $\delta=25$ & $\delta=40$ & $\delta=10$ & $\delta=25$ & $\delta=40$ \\
\hline 1 & 0.399 & $\mathbf{0 . 3 8 0}$ & 0.448 & 0.387 & 0.390 & 0.383 & 0.475 & 0.460 & 0.440 \\
2 & 0.327 & 0.303 & 0.294 & 0.310 & 0.308 & $\mathbf{0 . 2 6 3}$ & 0.344 & 0.328 & 0.326 \\
4 & 0.272 & 0.245 & 0.244 & 0.274 & 0.242 & $\mathbf{0 . 2 0 9}$ & 0.241 & 0.236 & 0.248 \\
8 & 0.198 & 0.170 & 0.165 & 0.213 & 0.193 & $\mathbf{0 . 1 6 2}$ & 0.183 & 0.214 & 0.198 \\
\hline
\end{tabular}

\subsection{Comparison of the algorithms ILS-SP, $\mathrm{HGS}^{r}$, and $\mathrm{POP}^{1}$ over 32 hours}

Figure 3 and Table 2 show the gap convergence curves for $\mathrm{HGS}^{r}$ and $\mathrm{POP}^{1}$ over the horizon of 32 hours.

Table 2: Average gap (\%) of $\mathrm{HGS}^{r}$ and $\mathrm{POP}^{1}$ executions at different times.

\begin{tabular}{|c|c|c|c|c|c|c|}
\hline Instances & Time $(\mathrm{h})$ & $\mathrm{HGS}^{r}$ & $\mathrm{POP}_{0.01}^{1}$ & $\mathrm{POP}_{0.125}^{1}$ & $\mathrm{POP}_{0.5}^{1}$ & $\mathrm{POP}_{2}^{1}$ \\
\hline \multirow{10}{*}{ All } & 0.01 & 1.996 & 1.996 & - & - & - \\
\hline & 0.125 & 0.836 & 1.180 & 0.836 & - & - \\
\hline & 0.25 & 0.626 & 0.931 & 0.564 & - & - \\
\hline & 0.5 & 0.484 & 0.708 & 0.457 & 0.484 & - \\
\hline & 1 & 0.396 & 0.579 & 0.355 & 0.317 & - \\
\hline & 2 & 0.330 & 0.420 & 0.271 & 0.240 & 0.330 \\
\hline & 4 & 0.283 & 0.293 & 0.206 & 0.182 & 0.171 \\
\hline & 8 & 0.236 & 0.201 & 0.164 & 0.138 & 0.126 \\
\hline & 16 & 0.210 & 0.137 & 0.117 & 0.099 & 0.090 \\
\hline & 32 & 0.184 & 0.091 & 0.084 & 0.076 & 0.064 \\
\hline \multirow{10}{*}{$\mathrm{X}_{S}$} & 0.01 & 1.790 & 1.790 & - & - & - \\
\hline & 0.125 & 0.704 & 0.754 & 0.704 & - & - \\
\hline & 0.25 & 0.547 & 0.524 & 0.398 & - & - \\
\hline & 0.5 & 0.425 & 0.350 & 0.303 & 0.425 & - \\
\hline & 1 & 0.345 & 0.268 & 0.228 & 0.232 & - \\
\hline & 2 & 0.298 & 0.196 & 0.163 & 0.178 & 0.298 \\
\hline & 4 & 0.262 & 0.101 & 0.114 & 0.131 & 0.108 \\
\hline & 8 & 0.196 & 0.058 & 0.069 & 0.084 & 0.081 \\
\hline & 16 & 0.176 & 0.039 & 0.048 & 0.056 & 0.055 \\
\hline & 32 & 0.162 & 0.017 & 0.022 & 0.036 & 0.037 \\
\hline \multirow{10}{*}{$\mathrm{X}_{L}$} & 0.01 & 2.209 & 2.209 & - & - & - \\
\hline & 0.125 & 0.973 & 1.621 & 0.973 & - & - \\
\hline & 0.25 & 0.708 & 1.354 & 0.735 & - & - \\
\hline & 0.5 & 0.546 & 1.080 & 0.617 & 0.546 & - \\
\hline & 1 & 0.448 & 0.902 & 0.487 & 0.404 & - \\
\hline & 2 & 0.363 & 0.652 & 0.383 & 0.305 & 0.363 \\
\hline & 4 & 0.306 & 0.492 & 0.301 & 0.235 & 0.237 \\
\hline & 8 & 0.278 & 0.349 & 0.263 & 0.193 & 0.173 \\
\hline & 16 & 0.244 & 0.238 & 0.190 & 0.144 & 0.127 \\
\hline & 32 & 0.206 & 0.167 & 0.149 & 0.117 & 0.092 \\
\hline
\end{tabular}

The performance of $\mathrm{POP}_{0.01}^{1}$ deserves a separate analysis. It illustrates the behavior of POP as an "almost stand-alone" heuristic, starting from a medium quality solution. Such solutions can be rapidly obtained by any modern metaheuristic for the CVRP. The initial solutions provided by running $\mathrm{HGS}^{r}$ for 36 seconds have an average gap of about $2 \%$ from the BKS.

- The performance of $\mathrm{POP}_{0.01}^{1}$ on instances with shorter routes (set $\mathrm{X}_{S}$ ) is very good. After 900 seconds, it already provides solutions that are significantly better than those from $\mathrm{HGS}^{r}$. After 4 hours, it is also consistently better than $\mathrm{POP}_{0.125}^{1}, \mathrm{POP}_{0.5}^{1}$, and $\mathrm{POP}_{2}^{1}$ and reaches the excellent average gap of $0.017 \%$ in 32 hours. It is quite interesting to note that the final gap after 32 hours obtained by $\mathrm{POP}_{t}^{1}$ on instances $\mathrm{X}_{S}$ gets worse as $t$ increases. It seems that worse initial solutions used in $\mathrm{POP}_{0.01}^{1}$ are still flexible enough to be transformed into good final solutions by the POP local search mechanism. On the other hand, the much better initial solutions used by $\mathrm{POP}_{0.5}^{1}$, and $\mathrm{POP}_{2}^{1}$ seem to be biased towards certain local minima that may not be so globally good.

- On the other hand, $\mathrm{POP}_{0.01}^{1}$ performs poorly on instances with long routes (set $\mathrm{X}_{L}$ ). It 

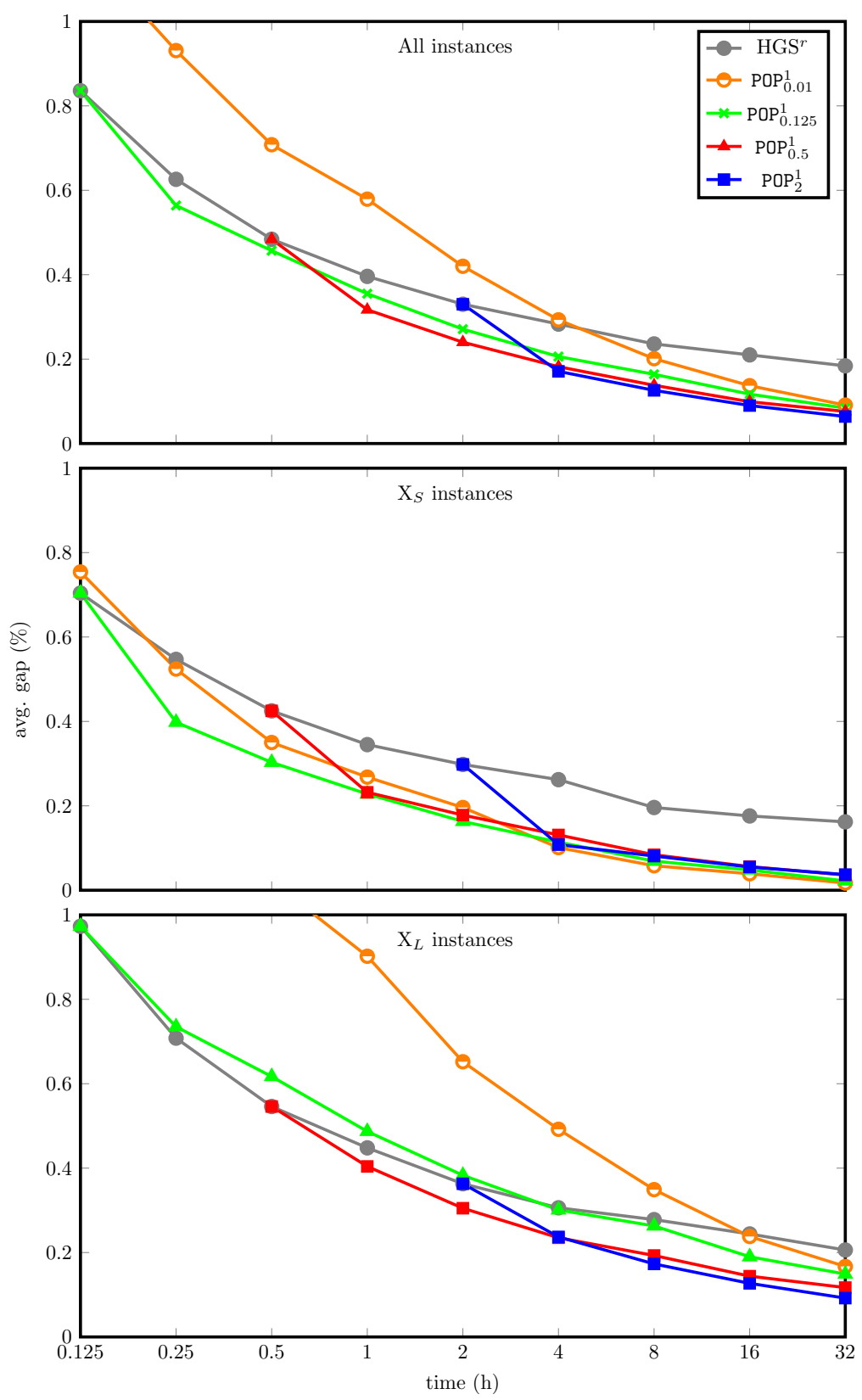

Figure 3: Convergence curves of $\mathrm{POP}^{1}$ and $\mathrm{HGS}^{r}$. 
Table 3: Best solutions found by ILS-SP, $\mathrm{HGS}^{r}$, and $\mathrm{POP}^{1}$ after 32 hours.

\begin{tabular}{|c|c|c|c|c|c|c|c|}
\hline Instance & $\mathrm{BKS}$ & ILS-SP & $\mathrm{HGS}^{r}$ & $\mathrm{POP}_{0.01}^{1}$ & $\mathrm{POP}_{0}^{1}$ & $\mathrm{POP}_{0.5}^{1}$ & $\mathrm{POP}_{2}^{1}$ \\
\hline X-n303-k21 & 21736 & 21840 & 21739 & 21863 & 21837 & 21750 & 21751 \\
\hline X-n308-k13 & 25859 & 25881 & 25861 & 25876 & 25876 & 25876 & 25862 \\
\hline X-n313-k71 & 94044 & 94105 & 94046 & 94053 & 94053 & 94046 & 94046 \\
\hline X-n317-k53 & $78355^{*}$ & 78355 & 78355 & 78355 & 78355 & 78355 & 78355 \\
\hline X-n322-k28 & $29834^{*}$ & 29872 & 29848 & 29887 & 29887 & 29887 & 29880 \\
\hline X-n327-k20 & 27532 & 27743 & 27555 & 27573 & 27576 & 27573 & 27576 \\
\hline X-n331-k15 & $31102^{*}$ & 31108 & 31103 & 31103 & 31103 & 31103 & 31103 \\
\hline X-n336-k84 & 139135 & 139253 & 139210 & 139164 & 139111 & 139175 & 139125 \\
\hline X-n344-k43 & 42056 & 42096 & 42069 & 42055 & 42050 & 42050 & 42056 \\
\hline X-n351-k40 & 25919 & 26131 & 25935 & 25896 & 25896 & 25896 & 25896 \\
\hline X-n359-k29 & 51505 & 51997 & 51521 & 51583 & 51583 & 51583 & 51505 \\
\hline X-n367-k17 & 22814 & 22912 & 22814 & 22814 & 22821 & 22821 & 22814 \\
\hline X-n376-k94 & $147713^{*}$ & 147713 & 147713 & 147713 & 147713 & 147713 & 147713 \\
\hline X-n384-k52 & 65941 & 66382 & 66048 & 65941 & 65999 & 65956 & 65947 \\
\hline X-n393-k38 & $38260^{*}$ & 38273 & 38260 & 38260 & 38260 & 38260 & 38260 \\
\hline $\mathrm{X}-\mathrm{n} 401-\mathrm{k} 29$ & 66163 & 66614 & 66222 & 66181 & 66220 & 66257 & 66156 \\
\hline X-n411-k19 & 19718 & 19811 & 19717 & 19712 & 19718 & 19712 & 19712 \\
\hline X-n420-k130 & $107798^{*}$ & 107798 & 107813 & 107798 & 107798 & 107798 & 107798 \\
\hline X-n429-k61 & 65449 & 65759 & 65489 & 65527 & 65467 & 65467 & 65455 \\
\hline X-n439-k37 & $36391^{*}$ & 36402 & 36395 & 36395 & 36395 & 36395 & 36395 \\
\hline $\mathrm{X}-\mathrm{n} 449-\mathrm{k} 29$ & 55233 & 56131 & 55336 & 55332 & 55236 & 55259 & 55258 \\
\hline X-n459-k26 & 24139 & 24421 & 24184 & 24193 & 24208 & 24209 & 24160 \\
\hline X-n469-k138 & $221824^{*}$ & 221940 & 222203 & 221824 & 221824 & 221824 & 221824 \\
\hline $\mathrm{X}-\mathrm{n} 480-\mathrm{k} 70$ & 89458 & 89821 & 89542 & 89449 & 89449 & 89449 & 89449 \\
\hline $1-\mathrm{k} 59$ & 66510 & 67128 & 66633 & 66555 & 66539 & 66572 & 66514 \\
\hline X-n502-k39 & 69230 & 69315 & 69254 & 69232 & 69232 & 69232 & 69232 \\
\hline X-n513-k21 & 24201 & 24275 & 24201 & 24248 & 24249 & 24201 & 24201 \\
\hline $\mathrm{X}-\mathrm{n} 524-\mathrm{k} 153$ & $154593^{*}$ & 154698 & 154774 & 154593 & 154593 & 154593 & 154593 \\
\hline X-n536-k96 & 94921 & 95697 & 95059 & 94948 & 94915 & 95205 & 95205 \\
\hline X-n548-k50 & $86700^{*}$ & 86710 & 86737 & 86701 & 86701 & 86701 & 86701 \\
\hline X-n561-k42 & 42717 & 43016 & 42744 & 42758 & 42773 & 42758 & 42758 \\
\hline $\mathrm{X}-\mathrm{n} 573-\mathrm{k} 30$ & 50673 & 51074 & 50782 & 50807 & 50882 & 50742 & 50735 \\
\hline X-n586-k159 & 190423 & 190767 & 190581 & 190365 & 190340 & 190375 & 190379 \\
\hline X-n599-k92 & 108489 & 109147 & 108781 & 108498 & 108558 & 108517 & 108462 \\
\hline $3-\mathrm{k} 62$ & 59535 & 60318 & 59671 & 59561 & 59544 & 59606 & 59656 \\
\hline$-\mathrm{k} 43$ & 62164 & 62762 & 62369 & 62182 & 62213 & 62245 & 62266 \\
\hline X-n641-k35 & 63694 & 64449 & 64019 & 63773 & 63989 & 63919 & 63863 \\
\hline X-n655-k131 & $106780^{*}$ & 106780 & 106810 & 106780 & 106780 & 106780 & 106780 \\
\hline X-n670-k130 & 146332 & 147286 & 147144 & 146346 & 146340 & 146411 & 146461 \\
\hline X-n685-k75 & 68205 & 68682 & 68436 & 68260 & 68315 & 68318 & 68354 \\
\hline X-n701-k44 & 81934 & 82907 & 82310 & 82085 & 81984 & 81970 & 82021 \\
\hline X-n716-k35 & 43412 & 44091 & 43572 & 43443 & 43491 & 43498 & 43489 \\
\hline X-n733-k159 & 136250 & 136900 & 136365 & 136245 & 136237 & 136278 & 136223 \\
\hline X-n749-k98 & 77365 & 78177 & 77706 & 77380 & 77399 & 77360 & 77342 \\
\hline X-n766-k71 & 114454 & 115413 & 114701 & 114573 & 114707 & 114640 & 114682 \\
\hline $\mathrm{X}-\mathrm{n} 783-\mathrm{k} 48$ & 72445 & 73627 & 72809 & 72696 & 72592 & 72605 & 72704 \\
\hline X-n801-k40 & 3305 & 73939 & 73548 & 73446 & 73445 & 73368 & 73362 \\
\hline X-n819-k171 & 158247 & 159249 & 158696 & 158128 & 158191 & 158222 & 158211 \\
\hline $\mathrm{X}-\mathrm{n} 837-\mathrm{k} 142$ & 193810 & 194901 & 194264 & 193820 & 193793 & 193800 & 193822 \\
\hline X-n856-k95 & 88965 & 89143 & 89062 & 89030 & 89030 & 89030 & 89030 \\
\hline X-n876-k59 & 99299 & 100357 & 99748 & 99583 & 99437 & 99479 & 99428 \\
\hline $\mathrm{X}-\mathrm{n} 895-\mathrm{k} 37$ & 53860 & 54777 & 54266 & 54112 & 54080 & 54125 & 54045 \\
\hline X-n916-k207 & 329247 & 330773 & 329902 & 329305 & 329213 & 329305 & 329289 \\
\hline X-n936-k151 & 132725 & 134564 & 133440 & 132859 & 132882 & 132863 & 132942 \\
\hline X-n957-k87 & 85465 & 85887 & 85633 & 85485 & 85468 & 85492 & 85473 \\
\hline X-n979-k58 & 118987 & 120015 & 119339 & 119430 & 119059 & 119073 & 119040 \\
\hline X-n1001-k43 & 72359 & 73810 & 72766 & 72714 & 72506 & 72460 & 72486 \\
\hline Avg. gap (\%) & & 0.629 & 0.184 & 0.091 & 0.084 & 0.076 & 0.064 \\
\hline Median gap (\%) & & 0.607 & 0.117 & 0.027 & 0.031 & 0.032 & 0.009 \\
\hline Avg. gap (\%) in $\mathrm{X}_{S}$ & & 0.468 & 0.162 & 0.017 & 0.022 & 0.036 & 0.037 \\
\hline Median gap (\%) in $\mathrm{X}_{S}$ & & 0.474 & 0.117 & 0.005 & 0.000 & 0.002 & 0.000 \\
\hline Avg. gap (\%) in $\mathrm{X}_{L}$ & & 0.796 & 0.206 & 0.167 & 0.149 & 0.117 & 0.092 \\
\hline Median gap (\%) in $\mathrm{X}_{L}$ & & 0.779 & 0.138 & 0.150 & 0.135 & 0.091 & 0.073 \\
\hline
\end{tabular}

takes 4 hours to obtain an average gap of $0.492 \%$, and it reaches the performance of HGS $^{r}$ only after 16 hours. It is also consistently worse than $\mathrm{POP}_{t}^{1}$, for $t \in\{0.125,0.5,2\}$.

The variants $\mathrm{POP}_{0.125}^{1}, \mathrm{POP}_{0.5}^{1}$, and $\mathrm{POP}_{2}^{1}$ have a more robust performance. When the complete instance set $\mathrm{X}$ is considered, all of them are consistently better than $\mathrm{HGS}^{r}$ alone (i.e., after POP starts, their average gaps are smaller at all times). This is also true when $\mathrm{X}_{S}$ and $\mathrm{X}_{L}$ instances are considered separately. The only exception is the variant $\mathrm{POP}_{0.125}^{1}$, which requires four hours to overcome $\mathrm{HGS}^{r}$ on $\mathrm{X}_{L}$ instances.

Table 3 reports the best solutions found by the algorithms ILS-SP, $\mathrm{HGS}^{r}$, and $\mathrm{POP}^{1}$ in 32 
hours. BKSs marked with $\mathrm{a}^{*}$ are proven optimal solutions. Solutions marked in bold are improvements over the BKSs. The variant $\mathrm{POP}_{2}^{1}$ achieved the best average and median final gaps, with the exception of the average gap for instances $\mathrm{X}_{S}$, where it is worse than the variants $\mathrm{POP}_{0.01}^{1}, \mathrm{POP}_{0.125}^{1}$, and $\mathrm{POP}_{0.5}^{1}$.

\subsection{Comparison of the algorithms HGS20 and $\mathrm{POP}^{2}$ over 32 hours}

When the work described in this article was already advanced, we were told ${ }^{2}$ about the existence of a new implementation of HGS. The new version, specialized to CVRP, is faster and includes one additional neighborhood called SWAP*. We will refer to that yet unpublished algorithm as HGS20. In fact, the performance of HGS20 is much superior to $\mathrm{HGS}^{r}$, and thus it can definitely be considered as a state-of-the-art metaheuristic for CVRP. In this section, we test if POP can still improve HGS20 solutions.

On September 17, 2020, Thibaut Vidal kindly sent us the detailed results of ten 20-hour runs of the algorithm HGS20 on each of the X instances. Those runs are performed on Intel Xeon Gold $6148 @ 2.40 \mathrm{GHz}$ processors (PassMark single thread rating 2056) that are roughly equivalent to our processors (PassMark single thread rating 1840). Moreover, we have also received the solutions obtained after $0.125,0.5$, and 2 hours. Thus, we use them as initial solutions in the variant $\mathrm{POP}_{t}^{2}$. Figure 4 depicts the performance of the HGS20 and the variants POP ${ }^{2}$ over 32 hours (note that HGS stops at 20 hours). We now analyze these results.

- For each running time, HGS20 obtains solutions with about half of the average gap of the solutions obtained by $\mathrm{HGS}^{r}$, which is a remarkable improvement.

- Considering all X instances, the variant $\mathrm{POP}_{0.125}^{2}$ is consistently worse than HGS20 alone, producing inferior solutions for all times.

- Considering all $\mathrm{X}$ instances, the variant $\mathrm{POP}_{0.5}^{2}$ is slightly better than the HGS20 alone. On $\mathrm{X}_{L}$ instances, it only starts to be better at 16 hours.

- Finally, the variant $\mathrm{POP}_{2}^{2}$ is clearly better than the HSG20 alone, even on $\mathrm{X}_{L}$ instances. This indicates that the proposed approach POP is indeed powerful. It is able to improve solutions obtained by a highly performing metaheuristic, at least in long runs (more than 2 hours).

Table 5 reports instance-by-instance statistics on the solutions found by the HGS20 (after 20 hours) and the variants POP ${ }^{2}$ (after 20 and 32 hours). For the HGS20, the average cost and the best cost among ten runs are provided. For the variant $\mathrm{POP}_{2}^{2}$, we give the average cost and the best cost for three runs. We did not have computational resources for running each instance ten times. In order to provide a direct comparison between methods, we also computed the average cost and the best cost of the first three runs of the HGS20.

It is obvious that one can always obtain better solutions by performing multiple runs of any randomized method and picking the best one. But it is still interesting to note that the variant $\mathrm{POP}_{2}^{2}$ seems to be particularly well suited for performing multiple runs. In fact, for this variant, the average gap for a single 32-hour run is $0.042 \%$, while the average gap for the best of only three runs decreases to $0.018 \%$, a very substantial decrease. For the instances in the set $\mathrm{X}_{S}$, the approach produces a remarkable gap of $-0.001 \%$.

\footnotetext{
${ }^{2}$ Personal communication from Thibaut Vidal.
} 


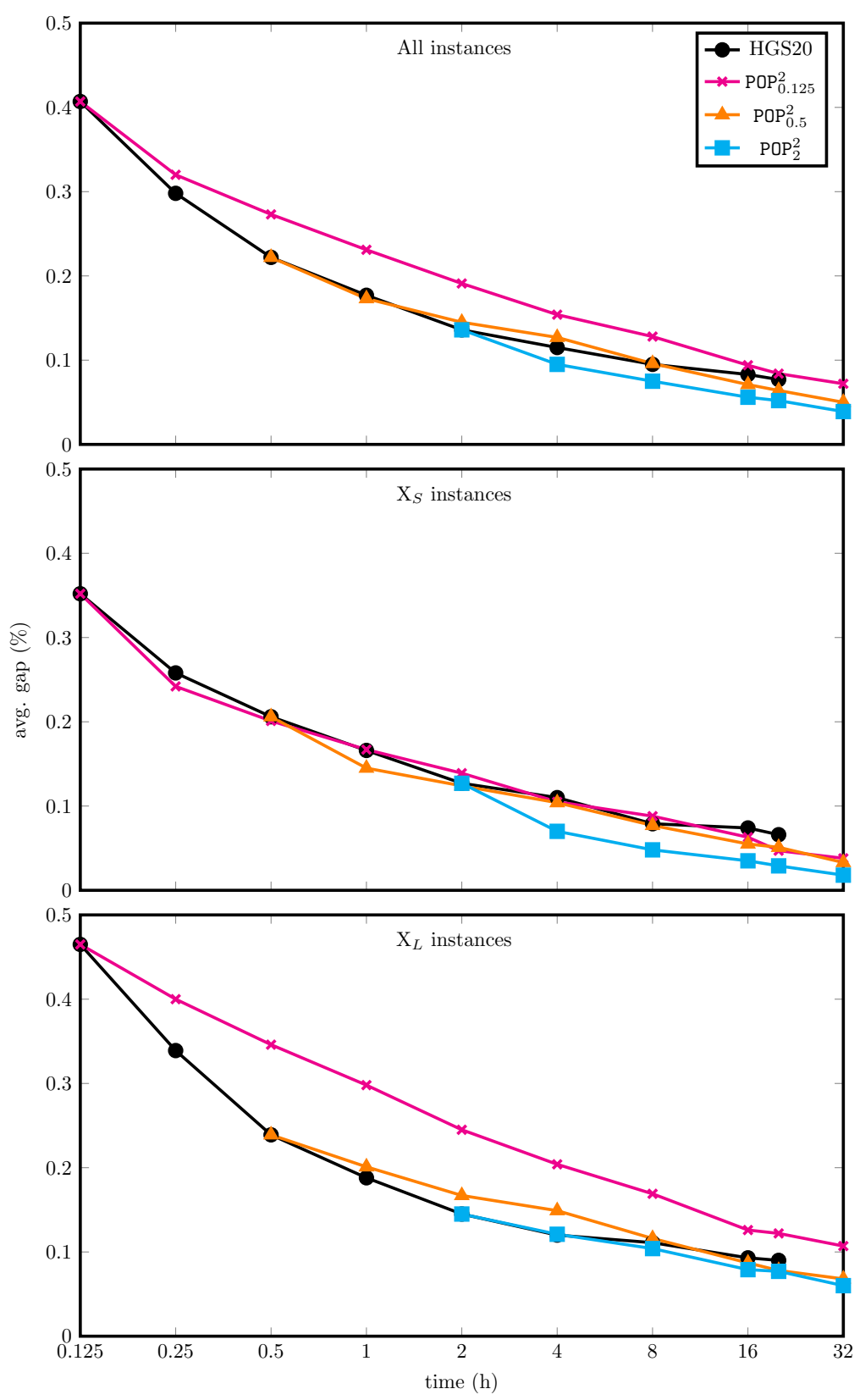

Figure 4: Convergence curves of $\mathrm{POP}^{2}$ and HGS20. 
Table 4: Average gap (\%) of HGS20 and POP ${ }^{2}$ executions at different times.

\begin{tabular}{|c|c|c|c|c|c|}
\hline Instances & Time (h) & HGS20 & $\mathrm{POP}_{0.125}^{2}$ & $\mathrm{POP}_{0.5}^{2}$ & $\mathrm{POP}_{2}^{2}$ \\
\hline \multirow{10}{*}{ All } & 0.125 & 0.407 & 0.407 & - & - \\
\hline & 0.25 & 0.298 & 0.320 & - & - \\
\hline & 0.5 & 0.222 & 0.273 & 0.222 & - \\
\hline & 1 & 0.177 & 0.231 & 0.173 & - \\
\hline & 2 & 0.136 & 0.191 & 0.145 & 0.136 \\
\hline & 4 & 0.115 & 0.154 & 0.127 & 0.095 \\
\hline & 8 & 0.095 & 0.128 & 0.096 & 0.075 \\
\hline & 16 & 0.083 & 0.094 & 0.071 & 0.056 \\
\hline & 20 & 0.077 & 0.084 & 0.064 & 0.052 \\
\hline & 32 & - & 0.072 & 0.050 & 0.039 \\
\hline \multirow{10}{*}{$\mathrm{X}_{S}$} & 0.125 & 0.352 & 0.352 & - & - \\
\hline & 0.25 & 0.258 & 0.242 & - & - \\
\hline & 0.5 & 0.206 & 0.201 & 0.206 & - \\
\hline & 1 & 0.166 & 0.167 & 0.145 & - \\
\hline & 2 & 0.127 & 0.139 & 0.124 & 0.127 \\
\hline & 4 & 0.110 & 0.105 & 0.104 & 0.070 \\
\hline & 8 & 0.079 & 0.088 & 0.077 & 0.048 \\
\hline & 16 & 0.074 & 0.063 & 0.055 & 0.035 \\
\hline & 20 & 0.066 & 0.047 & 0.051 & 0.029 \\
\hline & 32 & - & 0.038 & 0.033 & 0.018 \\
\hline \multirow{10}{*}{$\mathrm{X}_{L}$} & 0.125 & 0.465 & 0.465 & - & - \\
\hline & 0.25 & 0.339 & 0.400 & - & - \\
\hline & 0.5 & 0.239 & 0.346 & 0.239 & - \\
\hline & 1 & 0.188 & 0.298 & 0.201 & - \\
\hline & 2 & 0.145 & 0.245 & 0.167 & 0.145 \\
\hline & 4 & 0.120 & 0.204 & 0.149 & 0.121 \\
\hline & 8 & 0.111 & 0.169 & 0.116 & 0.104 \\
\hline & 16 & 0.093 & 0.126 & 0.087 & 0.079 \\
\hline & 20 & 0.090 & 0.122 & 0.078 & 0.077 \\
\hline & 32 & - & 0.107 & 0.068 & 0.060 \\
\hline
\end{tabular}


Table 5: Detailed statistics for HGS20 and POP2 2 . Best gaps for 20 hours are underlined.

\begin{tabular}{|c|c|c|c|c|c|c|c|c|c|}
\hline \multirow{2}{*}{ Instance } & \multirow{2}{*}{ BKS } & \multicolumn{4}{|c|}{$\begin{array}{c}\text { HGS20 } \\
20 \text { hours }\end{array}$} & \multicolumn{4}{|c|}{$\mathrm{POP}_{2}^{2}$} \\
\hline & & avg. cost $(10 \times)$ & best $(10 \times)$ & avg cost $(3 \times)$ & best $(3 \times)$ & avg cost $(3 \times)$ & best $(3 \times)$ & avg cost $(3 \times)$ & best $(3 \times)$ \\
\hline X-n303-k21 & 21736 & 21737.4 & 21736 & 21737.3 & 21736 & 21738.0 & 21738 & 21738.0 & 21738 \\
\hline X-n308-k13 & 25859 & 25859.0 & 25859 & 25859.0 & 25859 & 25859.7 & 25859 & 25859.7 & 25859 \\
\hline X-n317-k53 & $78355^{*}$ & 78355.0 & 78355 & 78355.0 & 78355 & 78355.0 & 78355 & 78355.0 & 78355 \\
\hline X-n322-k28 & $29834^{*}$ & 29834.0 & 29834 & 29834.0 & 29834 & 29834.0 & 29834 & 29834.0 & 29834 \\
\hline X-n327-k20 & 27532 & 27532.0 & 27532 & 27532.0 & 27532 & 27532.0 & 27532 & 27532.0 & 27532 \\
\hline X-n331-k15 & $31102^{*}$ & 31102.0 & 31102 & 31102.0 & 31102 & 31102.3 & 31102 & 31102.3 & 31102 \\
\hline X-n351-k40 & 25919 & 25925.2 & 25909 & 25917.3 & 25909 & 25903.7 & 25896 & 25903.7 & 25896 \\
\hline X-n359-k29 & 51505 & 51535.2 & 51513 & 51534.0 & 51513 & 51518.7 & 51505 & 51518.7 & 51505 \\
\hline X-n367-k17 & 22814 & 22814.0 & 22814 & 22814.0 & 22814 & 22814.0 & 22814 & 22814.0 & 22814 \\
\hline X-n376-k94 & $147713^{*}$ & 147713.0 & 147713 & 147713.0 & 147713 & 147713.0 & 147713 & 147713.0 & 147713 \\
\hline X-n384-k52 & 65941 & 65977.1 & 65957 & 65979.3 & 65978 & 65971.0 & 65941 & 65971.0 & 65941 \\
\hline X-n393-k38 & $38260^{*}$ & 38260.0 & 38260 & 38260.0 & 38260 & 38260.0 & 38260 & 38260.0 & 38260 \\
\hline X-n401-k29 & 66163 & 66196.9 & 66180 & 66203.7 & 66192 & 66188.7 & 66180 & 66185.0 & 66178 \\
\hline X-n459-k26 & 24139 & 24140.1 & 24139 & 24141.0 & 24139 & 24157.3 & 24139 & 24157.3 & 24139 \\
\hline X-n469-k138 & $221824^{*}$ & 221939.6 & 221848 & 221936.7 & 221855 & 221839.3 & 221824 & 221839.3 & 221824 \\
\hline X-n480-k70 & 89458 & 89459.2 & 89457 & 89461.3 & 89457 & 89457.0 & 89449 & 89449.0 & 89449 \\
\hline X-n491-k59 & 66510 & 66561.2 & 66521 & 66560.3 & 66521 & 66520.7 & 66489 & 66520.7 & 66489 \\
\hline X-n502-k39 & 69230 & 69228.5 & 69227 & 69229.3 & 69228 & 69226.0 & 69226 & 69226.0 & 69226 \\
\hline X-n513-k21 & 24201 & 24201.0 & 24201 & 24201.0 & 24201 & 24201.0 & 24201 & 24201.0 & 24201 \\
\hline X-n524-k153 & $154593^{*}$ & 154605.0 & 154605 & 154605.0 & 154605 & 154593.0 & 154593 & 154593.0 & 154593 \\
\hline X-n536-k96 & 94921 & 94991.5 & 94940 & 94991.0 & 94972 & 94948.0 & 94915 & 94943.0 & 94915 \\
\hline X-n548-k50 & $86700^{*}$ & 86710.0 & 86704 & 86706.0 & 86704 & 86700.7 & 86700 & 86700.7 & 86700 \\
\hline X-n561-k42 & 42717 & 42720.7 & 42717 & 42720.3 & 42717 & 42717.0 & 42717 & 42717.0 & 42717 \\
\hline X-n573-k30 & 50673 & 50747.1 & 50736 & 50742.0 & 50739 & 50741.0 & 50739 & 50738.3 & 50733 \\
\hline X-n586-k159 & 190423 & 190398.9 & 190340 & 190422.0 & 190407 & 190359.7 & 190349 & 190337.0 & 190316 \\
\hline X-n599-k92 & 108489 & 108554.2 & 108490 & 108562.0 & 108518 & 108486.0 & 108457 & 108484.7 & 108453 \\
\hline X-n613-k62 & 59535 & 59619.0 & 59549 & 59636.0 & 59602 & 59586.7 & 59536 & 59582.0 & 59536 \\
\hline X-n627-k43 & 62164 & 62273.3 & 62241 & 62275.3 & 62264 & 62264.7 & 62224 & 62254.0 & 62223 \\
\hline
\end{tabular}


Table 5 - continued from previous page

\begin{tabular}{|c|c|c|c|c|c|c|c|c|c|}
\hline \multirow[b]{2}{*}{ Instance } & \multirow[b]{2}{*}{ BKS } & \multirow{2}{*}{\multicolumn{4}{|c|}{$\begin{array}{c}\text { HGS20 } \\
20 \text { hours }\end{array}$}} & \multicolumn{4}{|c|}{$\mathrm{POP}_{2}^{2}$} \\
\hline & & & & & & 20 hol & & 32 hol & \\
\hline X-n655-k131 & $106780^{*}$ & 106787.6 & 106780 & 106789.7 & 106786 & 106780.0 & 106780 & 106780.0 & 106780 \\
\hline X-n701-k44 & 81934 & 82107.6 & 81998 & 82152.0 & 82123 & 82115.3 & 82030 & 82080.0 & 82030 \\
\hline X-n716-k35 & 43412 & 43468.3 & 43446 & 43481.0 & 43460 & 43455.7 & 43445 & 43433.3 & 43409 \\
\hline X-n733-k159 & 136250 & 136306.9 & 136281 & 136304.0 & 136298 & 136213.7 & 136195 & 136213.7 & 136195 \\
\hline X-n783-k48 & 72445 & 72649.8 & 72550 & 72665.0 & 72620 & 72572.0 & 72524 & 72563.7 & 72515 \\
\hline X-n801-k40 & 73305 & 73377.2 & 73308 & 73366.7 & 73353 & 73387.3 & 73385 & 73349.0 & 73313 \\
\hline X-n819-k171 & 158247 & 158331.3 & 158263 & 158318.0 & 158263 & 158328.7 & 158298 & 158298.3 & 158225 \\
\hline X-n837-k142 & 193810 & 194023.3 & 193973 & 193985.0 & 193973 & 193822.0 & 193756 & 193813.7 & 193739 \\
\hline X-n856-k95 & 88965 & 88986.4 & 88966 & 88983.7 & 88966 & 88989.7 & 88989 & 88989.7 & 88989 \\
\hline X-n876-k59 & 99299 & 99557.8 & 99490 & 99540.0 & 99510 & 99447.0 & 99428 & 99419.7 & 99405 \\
\hline X-n895-k37 & 53860 & 54041.2 & 54007 & 54028.3 & 54007 & 54021.3 & 53969 & 54000.0 & 53960 \\
\hline X-n916-k207 & 329247 & 329565.5 & 329481 & 329552.7 & 329539 & 329325.0 & 329288 & 329304.0 & 329249 \\
\hline Median gap (\%) & & 0.047 & 0.008 & 0.045 & 0.014 & $\underline{0.016}$ & $\underline{0.000}$ & 0.011 & 0.000 \\
\hline Avg. gap (\%) in $\mathrm{X}_{S}$ & & 0.068 & 0.031 & 0.068 & 0.048 & $\underline{0.029}$ & $\underline{0.007}$ & 0.021 & -0.001 \\
\hline Median gap (\%) in $\mathrm{X}_{S}$ & & 0.042 & 0.008 & 0.040 & 0.010 & $\underline{0.009}$ & $\underline{0.000}$ & 0.002 & 0.000 \\
\hline Avg. gap (\%) in $\mathrm{X}_{L}$ & & 0.092 & 0.055 & 0.093 & 0.073 & $\underline{0.076}$ & $\underline{0.048}$ & 0.064 & 0.037 \\
\hline Median gap (\%) in $\mathrm{X}_{L}$ & & 0.049 & 0.007 & 0.051 & 0.026 & $\underline{0.038}$ & $\underline{0.018}$ & 0.034 & 0.010 \\
\hline
\end{tabular}




\subsection{Directly improving BKSs in CVRPLIB}

In the final experiment, we run algorithm POP using the BKS in CVRPLIB as the initial solution. Besides testing the open X instances, we also test very large instances with up to 30,000 customers in the XXL set (Arnold et al., 2019) and also the open instances in the Golden set (Golden et al., 1998). Table 6 presents the improved BKSs found by POP after 32 hours for X and Golden instances, and after 96 hours for the XXL instances.

Table 6: BKSs directly improved by POP

\begin{tabular}{lrr}
\hline Instance & BKS & Improved BKS \\
\hline X-n536-k96 & 94868 & $\mathbf{9 4 8 6 4}$ \\
X-n733-k159 & 136190 & $\mathbf{1 3 6 1 8 8}$ \\
X-n766-k71 & 114454 & $\mathbf{1 1 4 4 1 8}$ \\
X-n783-k48 & 72394 & $\mathbf{7 2 3 9 3}$ \\
X-n936-k151 & 132725 & $\mathbf{1 3 2 7 1 5}$ \\
X-n979-k58 & 118987 & $\mathbf{1 1 8 9 7 6}$ \\
X-n1001-k43 & 72359 & $\mathbf{7 2 3 5 5}$ \\
\hline Antwerp1 & 477306 & $\mathbf{4 7 7 2 7 7}$ \\
Brussels1 & 501854 & $\mathbf{5 0 1 7 7 1}$ \\
Flanders1 & 7241290 & $\mathbf{7 2 4 0 8 7 4}$ \\
Ghent1 & 469586 & $\mathbf{4 6 9 5 3 2}$ \\
Leuven1 & 192851 & $\mathbf{1 9 2 8 4 8}$ \\
\hline Golden_16 & 1611.70 & $\mathbf{1 6 1 1 . 2 8}$ \\
\hline
\end{tabular}

\section{Concluding remarks}

In this work, we propose POP, a POPMUSIC matheuristic for the classical and highly competitive CVRP. The algorithm is designed to improve a reasonably good initial solution given as an input. The results show that our approach outperforms one of the best published metaheuristics for the CVRP in medium and long runs. POP matheuristic is also competitive in long runs with a state-ofthe-art unpublished heuristic, which is specialized to the CVRP. The results are especially good for instances with relatively short routes. Moreover, several best known solutions were improved for literature instances with up to 20,000 customers. This shows a very good scalability of the approach.

POP matheuristic exploits a characteristic of the modern exact algorithms for vehicle routing problems, and in particular, those for the CVRP. If a tight upper bound on the optimum value is provided, those exact algorithms are usually capable of solving to optimality medium-size instances with up to 100-150 customers in a few minutes. Instances with less than 100 customers are usually solved in seconds. Thus, exact (or almost exact, the case of $\mathrm{BCP}_{H}$ ) approaches become competitive with the best heuristics for solving such instances. An important advantage of exact approaches is that they "know" when to stop after proving that an improving solution does not exist, whereas traditional heuristics do not possess that information.

POP has interesting features that may be explored in future works:

- It is very different from other existing and well-performing heuristics for the CVRP. This means that their strengths and weaknesses may be complementary. This opens possibilities for many types of hybridization. It could be something simple, like just determining the instance characteristics (besides route size) that make it more or less suited to POP, in order to decide which method should be applied. But it could be something deeper, a full integration where POP and some traditional metaheuristic could take turns on improving parts of a solution and exchange information. This seems to be quite a promising direction for research. 
- It is easy to implement, provided that an exact (or nearly exact) code for solving the subproblems is at hand. Given that VRPSolver branch-cut-and-price algorithm is available for academic use and can solve many routing variants other than CVRP, it is natural to try algorithms similar POP on those variants. Of course, there is no guarantee that a straightforward adaptation will obtain good results. Thus, there is room for research on extensions of POP that are more suited for other particular routing problems.

- It is naturally parallelizable. The current sequential version of POP may take a few hours to obtain high-quality solutions and can not be used in practical situations that require faster solutions. That limitation could be much reduced by solving several subproblems in parallel, a natural feature of any POPMUSIC approach. In fact, the larger the instance, the more parallelizable the method becomes.

The last remark is that the underlying implementation of the BCP solver used in POP was not changed by us other than by modifying external parameters. Thus, there is a large potential to improve the efficiency of POP by "going inside the black box". A property that may be exploited is that the subproblems to be solved are often very similar to some already solved subproblem. Keeping information from previous runs, like the generated columns and cuts, may accelerate the algorithm.

\section{Acknowledgements}

This study was financed in part by CAPES - Finance Code 001, by Capes PrInt UFF no 88881, by CNPq grant 313601/2018-6, and by FAPERJ grant E-26/202.887/2017.

This research was also supported by Programa Institucional de Internacionalização (PrInt) from CAPES as part of the project REMATCH (process number 88887.310261/2018-00).

The experiments presented in this paper were carried out using the PlaFRIM experimental testbed, supported by Inria, CNRS (LABRI and IMB), Université de Bordeaux, Bordeaux INP and Conseil Régional d'Aquitaine (see https://www.plafrim.fr/).

We would like to thank Anand Subramanian for kindly sending us his code.

We are grateful to Thibaut Vidal for kindly providing us the results of his new heuristic and for insightful discussions.

\section{References}

Luca Accorsi and Daniele Vigo. A fast and scalable heuristic for the solution of large-scale capacitated vehicle routing problems. Technical report, University of Bologna, 2020.

Claudia Archetti and M. Grazia Speranza. A survey on matheuristics for routing problems. EURO Journal on Computational Optimization, 2(4):223-246, Nov 2014. ISSN 2192-4414.

Florian Arnold and Kenneth Sörensen. Knowledge-guided local search for the vehicle routing problem. Computers \& Operations Research, 105:32 - 46, 2019. ISSN 0305-0548. doi: https: //doi.org/10.1016/j.cor.2019.01.002.

Florian Arnold, Michel Gendreau, and Kenneth Sörensen. Efficiently solving very large-scale routing problems. Computers $\& 3$ Operations Research, 107:32 - 42, 2019. ISSN 0305-0548. doi: https://doi.org/10.1016/j.cor.2019.03.006.

Roberto Baldacci, Nicos Christofides, and Aristide Mingozzi. An exact algorithm for the vehicle routing problem based on the set partitioning formulation with additional cuts. Mathematical Programming, 115(2):351-385, Oct 2008. ISSN 1436-4646. doi: 10.1007/s10107-007-0178-5. 
Roberto Baldacci, Aristide Mingozzi, and Roberto Roberti. New route relaxation and pricing strategies for the vehicle routing problem. Operations Research, 59(5):1269-1283, 2011. doi: 10.1287 /opre.1110.0975.

Teobaldo Bulhões, Guillaume Marques, Artur Pessoa, Eduardo Queiroga, Ruslan Sadykov, Eduardo Uchoa, and François Vanderbeck. VRPSolver: a branch-cut-and-price based exact solver for vehicle routing and some related problems. https://vrpsolver.math.u-bordeaux.fr/, 2020. Accessed: 2020-10-16.

Teobaldo Bulhões, Artur Pessoa, Fábio Protti, and Eduardo Uchoa. On the complete set packing and set partitioning polytopes: Properties and rank 1 facets. Operations Research Letters, 46 (4):389 - 392, 2018. ISSN 0167-6377. doi: https://doi.org/10.1016/j.orl.2018.04.006.

Jan Christiaens and Greet Vanden Berghe. Slack induction by string removals for vehicle routing problems. Transportation Science, 54(2):417-433, 2020.

Claudio Contardo and Rafael Martinelli. A new exact algorithm for the multi-depot vehicle routing problem under capacity and route length constraints. Discrete Optimization, 12:129 - 146, 2014. ISSN 1572-5286. doi: https://doi.org/10.1016/j.disopt.2014.03.001.

Luciano Costa, Claudio Contardo, and Guy Desaulniers. Exact branch-price-and-cut algorithms for vehicle routing. Transportation Science, 53(4):946-985, 2019.

G. B. Dantzig and J. H. Ramser. The truck dispatching problem. Management Science, 6(1): 80-91, 1959.

Bruce L. Golden, Edward A. Wasil, James P. Kelly, and I-Ming Chao. The Impact of Metaheuristics on Solving the Vehicle Routing Problem: Algorithms, Problem Sets, and Computational Results, pages 33-56. Springer US, Boston, MA, 1998. ISBN 978-1-4615-5755-5. doi: 10.1007/978-1-4615-5755-5_2.

Stefan Irnich, Guy Desaulniers, Jacques Desrosiers, and Ahmed Hadjar. Path-reduced costs for eliminating arcs in routing and scheduling. INFORMS Journal on Computing, 22(2):297-313, 2010.

Mads Jepsen, Bjørn Petersen, Simon Spoorendonk, and David Pisinger. Subset-row inequalities applied to the vehicle-routing problem with time windows. Operations Research, 56(2):497$511,2008$.

Laetitia Jourdan, Matthieu Basseur, and E-G Talbi. Hybridizing exact methods and metaheuristics: A taxonomy. European Journal of Operational Research, 199(3):620-629, 2009.

S. Kirkpatrick, C. D. Gelatt, and M. P. Vecchi. Optimization by simulated annealing. Science, 220(4598):671-680, 1983. ISSN 0036-8075. doi: 10.1126/science.220.4598.671.

Eduardo Lalla-Ruiz and Stefan Voß. A POPMUSIC approach for the multi-depot cumulative capacitated vehicle routing problem. Optimization Letters, 14(3):671-691, Apr 2020. ISSN 1862-4480.

G. Laporte and Y. Nobert. A branch and bound algorithm for the capacitated vehicle routing problem. Operations-Research-Spektrum, 5(2):77-85, Jun 1983. ISSN 1436-6304. doi: 10. $1007 / \mathrm{BF} 01720015$.

Gilbert Laporte, Stefan Ropke, and Thibaut Vidal. Chapter 4: Heuristics for the Vehicle Routing Problem, pages 87-116. 2014. doi: 10.1137/1.9781611973594.ch4.

Valeria Leggieri and Mohamed Haouari. A matheuristic for the asymmetric capacitated vehicle routing problem. Discrete Applied Mathematics, 234:139 - 150, 2018. ISSN 0166-218X. doi: https://doi.org/10.1016/j.dam.2016.03.019. Special Issue on the Ninth International Colloquium on Graphs and Optimization (GO IX), 2014. 
Helena Ramalhinho Lourenço, Olivier C Martin, and Thomas Stützle. Iterated local search: Framework and applications. In Handbook of metaheuristics, pages 129-168. Springer, 2019.

Jens Lysgaard. Cvrpsep: A package of separation routines for the capacitated vehicle routing problem. Tech. report, Aarhus University, Denmark, 2003.

A Ostertag, K F Doerner, R F Hartl, E D Taillard, and P Waelti. Popmusic for a real-world large-scale vehicle routing problem with time windows. Journal of the Operational Research Society, 60(7):934-943, 2009.

Diego Pecin, Artur Pessoa, Marcus Poggi, and Eduardo Uchoa. Improved branch-cut-and-price for capacitated vehicle routing. In Jon Lee and Jens Vygen, editors, Integer Programming and Combinatorial Optimization, pages 393-403, Cham, 2014. Springer International Publishing. ISBN 978-3-319-07557-0.

Diego Pecin, Artur Pessoa, Marcus Poggi, and Eduardo Uchoa. Improved branch-cut-and-price for capacitated vehicle routing. Mathematical Programming Computation, 9(1):61-100, Mar 2017a. ISSN 1867-2957.

Diego Pecin, Artur Pessoa, Marcus Poggi, Eduardo Uchoa, and Haroldo Santos. Limited memory rank-1 cuts for vehicle routing problems. Operations Research Letters, 45(3):206 - 209, $2017 \mathrm{~b}$. ISSN 0167-6377. doi: https://doi.org/10.1016/j.orl.2017.02.006.

Artur Pessoa, Eduardo Uchoa, and Marcus Poggi de Aragão. A robust branch-cut-and-price algorithm for the heterogeneous fleet vehicle routing problem. Networks: An International Journal, 54(4):167-177, 2009.

Artur Pessoa, Ruslan Sadykov, Eduardo Uchoa, and François Vanderbeck. A generic exact solver for vehicle routing and related problems. Mathematical Programming, 183(1):483-523, Sep 2020. ISSN 1436-4646. doi: 10.1007/s10107-020-01523-z.

Marcus Poggi and Eduardo Uchoa. Chapter 3: New exact algorithms for the capacitated vehicle routing problem. In Vehicle Routing: Problems, Methods, and Applications, Second Edition, pages 59-86. SIAM, 2014.

Ruslan Sadykov, Eduardo Uchoa, and Artur Pessoa. A bucket graph based labeling algorithm with application to vehicle routing. Transportation Science, Ahead of Print, 2020.

Anand Subramanian, Eduardo Uchoa, and Luiz Satoru Ochi. A hybrid algorithm for a class of vehicle routing problems. Computers $\&$ Operations Research, 40(10):2519 - 2531, 2013. ISSN 0305-0548. doi: https://doi.org/10.1016/j.cor.2013.01.013.

E. Taillard and K. Helsgaun. POPMUSIC for the travelling salesman problem. European Journal of Operational Research, 272(2):420 - 429, 2019. ISSN 0377-2217.

Éric D. Taillard and Stefan Voss. Popmusic - Partial Optimization Metaheuristic under Special Intensification Conditions, pages 613-629. Springer US, Boston, MA, 2002. ISBN 978-1-46151507-4.

Eduardo Uchoa, Diego Pecin, Artur Pessoa, Marcus Poggi, Thibaut Vidal, and Anand Subramanian. New benchmark instances for the capacitated vehicle routing problem. European Journal of Operational Research, 257(3):845 - 858, 2017. ISSN 0377-2217.

F. Vanderbeck, R. Sadykov, and I. Tahiri. BaPCod - a generic branch-and-price code. Available at https://realopt.bordeaux.inria.fr/?page_id=2, 2018.

Thibaut Vidal, Teodor Gabriel Crainic, Michel Gendreau, Nadia Lahrichi, and Walter Rei. A hybrid genetic algorithm for multidepot and periodic vehicle routing problems. Operations Research, 60(3):611-624, 2012. doi: 10.1287/opre.1120.1048. 


\section{A A representative small subset of $X$}

A minimum subset of the instances $\mathrm{X}$ which covers all the characteristics considered in Uchoa et al. (2017) is described below:

- Route size (interval for $n / K_{\min }$ ):

$-[3,5]:$ X-n469-k138

- (5,8]: X-n670-k130

- $(8,11]:$ X-n393-k38

- (11,14]: X-n561-k42

- (14,17]: X-n979-k58

$-(17,20]: X-n 801-k 40$

- (20, 25]: X-n716-k35

- Depot positioning:

- Random: X-n670-k130, X-n716-k35

- Center: X-n393-k38, X-n561-k42

- Corner: X-n469-k138, X-n801-k40, X-n979-k58

- Customers distribution:

- Random: X-n469-k138, X-n670-k130, X-n801-k40

- Clustered: X-n716-k35, X-n979-k58

- Random-clustered: X-n393-k38, X-n561-k42

- Customers demands:

- Unitary: X-n801-k40

- Small values, large CV ${ }^{3}$ : X-n561-k42

- Small values, small CV: X-n393-k38

- Large values, large CV: X-n716-k35

- Large values, small CV: X-n469-k138

- Depending on quadrant: X-n979-k58

- Many small values, few large values: X-n670-k130

Thus, the subset $\mathrm{X}_{R}$ is composed by: X-n393-k38, X-n469-k138, X-n561-k42, X-n670-k130, X-n716-k35, X-n801-k40, X-n979-k58. The reader is referred to Uchoa et al. (2017) for a detailed description of all characteristics.

\section{B Comparison of HGS and HGS ${ }^{r}$}

Figure 5 compares the performance of a single execution of HGS and $\mathrm{HGS}^{r}$ for all $X$ instances over 8 hours. The convergence curves of both algorithms report the average gap (considering the gap obtained for each instance) found at different times. The final average gaps obtained by HGS and $\mathrm{HGS}^{r}$ were $0.281 \%$ and $0.236 \%$, respectively.

${ }^{3}$ Coefficient of variation 


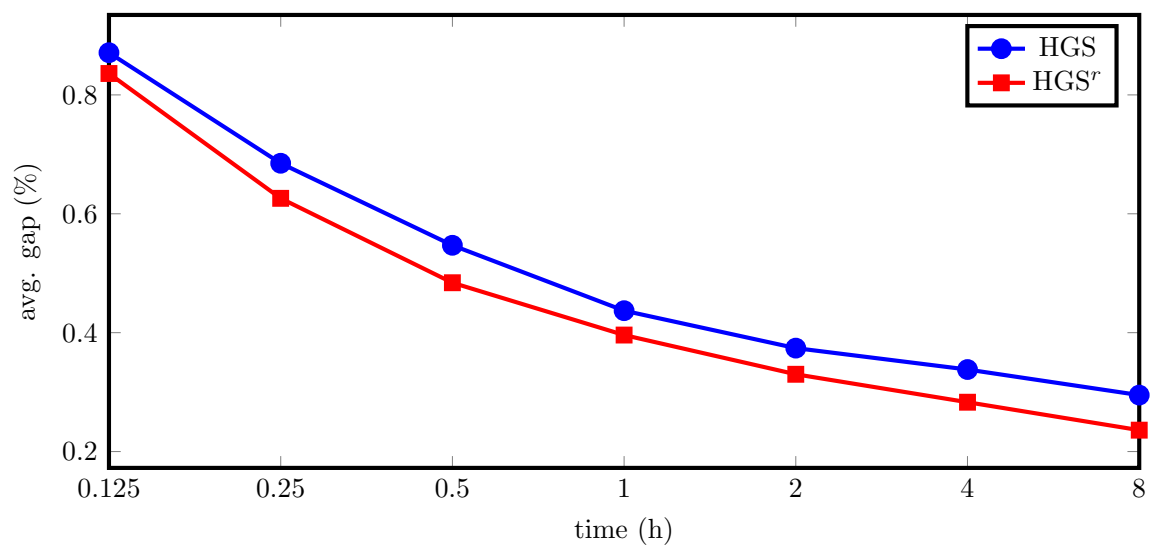

Figure 5: Comparison of HGS and $\mathrm{HGS}^{r}$ w.r.t. the convergence curve based on the average gap for all $X$ instances over 8 hours. The time axis is on a $\log _{2}$ scale. 


\section{VRPSolver Parameterizations}

Table 7 shows the default VRPSolver CVRP parameterization, as well as the changed parameterization in the version used to solve subproblems in POP. The reader is referred to the documentation of Bulhões et al. (2020) to obtain the description of each parameter. The parameters which have special notation indicated in the second column of Table 7 are also described in Pessoa et al. (2020). Default values for the last four parameters are not defined because these parameters are not active when the restricted master heuristic is not used. RCSPmaxNumOfEnumSolsForEndOfNodeMIP is a previously undocumented VRPSolver parameter. If the number of enumerated routes gets becomes smaller than RCSPmaxNumOfEnumSolutionsForMIP, at any point of a node solution, then the node is immediately solved by MIP.

Table 7: Default and used parameters of the VRPSolver CVRP application used in

\begin{tabular}{|c|c|c|c|}
\hline Parameter & Notation & Default value & Used value \\
\hline RCSPhardTimeThresholdInPricing & $\tau^{\text {hard }}$ & 25 secs & 8 secs \\
\hline RCSPstopCutGenTimeThresholdInPricing & $\tau^{\text {soft }}$ & 10 secs & 3 secs \\
\hline RCSPnumberOfBucketsPerVertex & $\tau^{\text {hard }}$ & 25 & 50 \\
\hline RCSPmaxNumOfLabelsInEnumeration & $\omega^{\text {labels }}$ & $5 \cdot 10^{6}$ & $3 \cdot 10^{5}$ \\
\hline RCSPmaxNum0fEnumeratedSolutions & $\omega^{\text {routes }}$ & $5 \cdot 10^{6}$ & $10^{6}$ \\
\hline RCSPmaxNumOfEnumSolutionsForMIP & $\omega^{\mathrm{MIP}}$ & $10^{4}$ & $5 \cdot 10^{3}$ \\
\hline RCSPmaxNum0fEnumSolsForEndOfNodeMIP & - & $10^{4}$ & $10^{4}$ \\
\hline RCSPuseBidirectionalSearch & $\phi^{\text {bidir }}$ & 2 & 1 \\
\hline RCSPrankOneCutsMemoryType & $\theta^{\mathrm{mem}}$ & 0 & 0 \\
\hline CutTailingOffThreshold & $\delta^{\text {gap }}$ & 0.015 & 0.03 \\
\hline StrongBranchingPhaseOneCandidatesNumber & $\zeta_{1}^{\text {num }}$ & 100 & 50 \\
\hline StrongBranchingPhaseOneTreeSizeEstimRatio & $\zeta_{1}^{\text {estim }}$ & 0.2 & 0.2 \\
\hline StrongBranchingPhaseTwoCandidatesNumber & $\zeta_{2}^{\text {num }}$ & 5 & 3 \\
\hline StrongBranchingPhaseTwoTreeSizeEstimRatio & $\zeta_{2}^{\text {estim }}$ & 0.02 & 0.02 \\
\hline MaxTimeForRestrictedMasterIpHeur & $\chi^{\mathrm{rm}}$ & -1 (off) & 40 \\
\hline CallFrequencyOfRestrictedMasterIpHeur & - & - & 1 \\
\hline MIPemphasisInRestrictedMasterIpHeur & - & - & 1 \\
\hline RCSPmaxNum0fLabels InHeurEnumeration & - & - & $10^{5}$ \\
\hline MaxNumEnumSolsInRestrictedMasterIpHeur & - & - & $10^{4}$ \\
\hline
\end{tabular}

Table 8 shows the additional parameters used for obtaining $\mathrm{BCP}_{H}$. While they reduce running times, the optimal solution of a subproblem may be missed.

Table 8: Additional parameters for obtaining $\mathrm{BCP}_{H}$

\begin{tabular}{cccc}
\hline Parameter & Notation & Default value & Used value \\
\hline GlobalTimeLimit & - & $\infty$ & 3600 secs \\
MaxNbOfBBtreeNodeTreated & - & $\infty$ & 10 \\
RCSPfalseGapFactor & - & 0 (off) & 3 \\
\hline
\end{tabular}

